\title{
Deep brain stimulation for treatment-refractory obsessive compulsive disorder: a systematic review
}

Sina Kohl ${ }^{1}$, Deva M Schönherr ${ }^{1}$, Judy Luigjes ${ }^{2}$, Damiaan Denys ${ }^{2,3}$, Ulf J Mueller ${ }^{4}$, Doris Lenartz ${ }^{5}$, Veerle Visser-Vandewalle $e^{5}$ and Jens Kuhn ${ }^{1 *}$

\begin{abstract}
Background: Obsessive-compulsive disorder is one of the most disabling of all psychiatric illnesses. Despite available pharmacological and psychotherapeutic treatments about $10 \%$ of patients remain severely affected and are considered treatment-refractory. For some of these patients deep brain stimulation offers an appropriate treatment method. The scope of this article is to review the published data and to compare different target structures and their effectiveness.

Methods: PubMed search, last update June 2013, was conducted using the terms "deep brain stimulation" and "obsessive compulsive disorder".

Results: In total 25 studies were found that reported five deep brain stimulation target structures to treat obsessive-compulsive disorder: the anterior limb of the internal capsule (five studies including 14 patients), nucleus accumbens (eight studies including 37 patients), ventral capsule/ventral striatum (four studies including 29 patients), subthalamic nucleus (five studies including 23 patients) and inferior thalamic peduncle (two studies including 6 patients). Despite the anatomical diversity, deep brain stimulation treatment results in similar response rates for the first four target structures. Inferior thalamic peduncle deep brain stimulation results in higher response rates but these results have to be interpreted with caution due to a very small number of cases. Procedure and device related adverse events are relatively low, as well as stimulation or therapy related side effects. Most stimulation related side effects are transient and decline after stimulation parameters have been changed.

Conclusion: Deep brain stimulation in treatment-refractory obsessive-compulsive disorder seems to be a relatively safe and promising treatment option. However, based on these studies no superior target structure could be identified. More research is needed to better understand mechanisms of action and response predictors that may help to develop a more personalized approach for these severely affected obsessive compulsive patients.
\end{abstract}

Keywords: Deep brain stimulation, Obsessive compulsive disorder, Treatment-refractory, Psychiatric surgery

\section{Background}

Obsessive-compulsive disorder (OCD) is one of the most disabling of all psychiatric illnesses [1] with a lifetime prevalence of $2 \%-3 \%$ and an early onset in adolescence or young adulthood [2]. Core symptoms of OCD are recurrent and intrusive thoughts and repetitive behaviours that are time consuming, cause remarkable distress and are often perceived as inappropriate. Even with the

\footnotetext{
* Correspondence: jens.kuhn@uk-koeln.de

'Department of Psychiatry and Psychotherapy, University of Cologne,

Kerpener Strasse 62, 50937 Cologne, Germany

Full list of author information is available at the end of the article
}

currently available treatment options, OCD has a tendency to chronicity and can have a devastating effect on occupational functioning, relationships and social activities. Besides cognitive-behavioural and psychodynamic models, neurobiological causes are considered to explain the complex symptomatology of this mental disorder. However, the detailed mechanisms underlying obsessions and compulsions are not completely understood. A huge amount of imaging findings -partially inconsistent- indicate that OCD pathology is correlated with dysfunctions of the cortico-striato-pallido-thalamo-cortical pathway [3] and a failure of ventral striatum inhibition [4]. In this 
context, two recent studies are of special importance, which hint at the dysregulation, i.e. pathological hyperconnectivity between cortical and striatal structures, as potential causal factors for OCD symptoms [5,6]. Still, specific treatments for OCD such as pharmacotherapy with serotonin reuptake inhibitors and cognitive behavioural therapy with exposure and response prevention provide only $40 \%$ to $60 \%$ symptom reduction in half of the patients, without having elucidated the exact mechanisms of action. Consequently, approximately $10 \%$ of patients remain severely affected and suffer from treatment-resistant obsessive-compulsive disorder (tr-OCD) [7].

Deep Brain Stimulation (DBS) is an innovative, neuromodulating treatment method, which was introduced in the late $1980 \mathrm{~s}$ in a patient suffering from severe tremor [8]. DBS affects the functioning of subcortical neuronal circuits by implanting electrodes in the brain using stereotactic coordinates. The electrodes discharge chronic high-frequency electrical pulses that alter the neuronal activity in the region of interest [9]. For a long time DBS was hypothesized to cause stimulation-induced functional lesioning but this view has been challenged in the last years. Rather it can be assumed that DBS unfolds very diverse neuromodulative effects dependent on target structure and disease. For example, research indicates influences on neurotransmitter release, oscillatory activity and inhibiting as well as activating network effects distal from the actual stimulation target [10]. For movement disorders, including Parkinson and essential tremor, DBS is an accepted and effective treatment option [11]. Because of its high effectiveness in neurological disorders, research also started to focus on the application of DBS for psychiatric disorders, e.g. OCD [12,13], Gilles de la Tourette syndrome [14,15], addiction disorders [16,17], and depression [18]. In 2009, DBS has been accepted in the US as a treatment option for tr-OCD patients by approving Medtronic reclaim approach by the US Food and Drug Association under the Human Device Exemption (see: http://www.accessdata.fda.gov/scripts/cdrh/cfdocs/ cftopic/pma/pma.cfm?num=H050003).

CE-certification by European public authorities followed shortly thereafter. For a small proportion of tr- OCD patients, DBS may be appropriate. The rationale behind DBS in OCD is based on the aforementioned neurobiological assumptions, namely that OCD is associated with hyperactivity and hyperconnectivity within this cortico-striatopallido-thalamo-cortical network and although the exact mechanism of DBS is unknown, it is hypothesized that DBS functionally overrides and modulates pathological hyperactivity in disturbed networks. Studies combining imaging and DBS are sparse, but it has been suggested that prefrontal activity as well as connectivity between the prefrontal cortex (PFC) and the nucleus accumbens (NAc) normalizes following DBS [19].
Since 1999 over 200 OCD patients have received experimental DBS. Five different target structures have been used for DBS: the anterior limb of the internal capsule (ALIC), nucleus accumbens (NAc), ventral capsule/ ventral striatum (VC/VS), subthalamic nucleus (STN) and inferior thalamic peduncle (ITP) (whether these five structures correspond to five targets will be discussed later). The scope of this article is to review the published data and to compare different target structures and their effectiveness.

\section{Methods}

For this purpose, we examined original published reports on studies about DBS for OCD. A PubMed search, last update 24th of June 2013, was conducted using the terms "deep brain stimulation" and "obsessive compulsive disorder". The PubMed search resulted in 279 articles (Figure 1). We scanned all abstracts that were available in either English or German language, and included those that were peer-reviewed, published, original articles on clinical trials of DBS for OCD. In addition we used a previously published review [20] and screened the lists of references of the relevant publications to complete our list of studies. To ensure correct data collection one researcher extracted the data and a second researcher independently checked the data extraction forms for accuracy and completeness. Finally, we included 25 articles in the present review. We summarized twenty-three articles that included information on Yale-Brown ObsessiveCompulsive Scale (Y-BOCS) scores in five tables arranged by target structure and calculated mean response rate and mean change in Y-BOCS score for each target location. To avoid biased results we ensured to not include duplicates. For that reason not all Y-BOCS score in the tables have been included in the quantitative analysis. All studies included in this review have been approved by the responsible local ethical commission. For the purpose of reviewing published literature no ethical approval is required by the guidelines of the ethics committee of the Medical Faculty of the University of Cologne. A review protocol is not available.

\section{Results}

\section{Anterior limb of internal capsule}

The ALIC is the part of the Internal Capsule that lies anterior to the genu, located between the head of the caudate nucleus and the lenticular nucleus. Its fibers connect the prefrontal cortex and the subcortical nuclei, specifically the dorsomedial thalamus. Outcomes of anterior capsulotomy for refractory OCD led to the selection of the ALIC as a target for DBS [22].

Nuttin and colleagues (1999) were the first to replace lesioning by high frequency DBS in OCD patients. They reported beneficial effects after bilateral stimulation of 


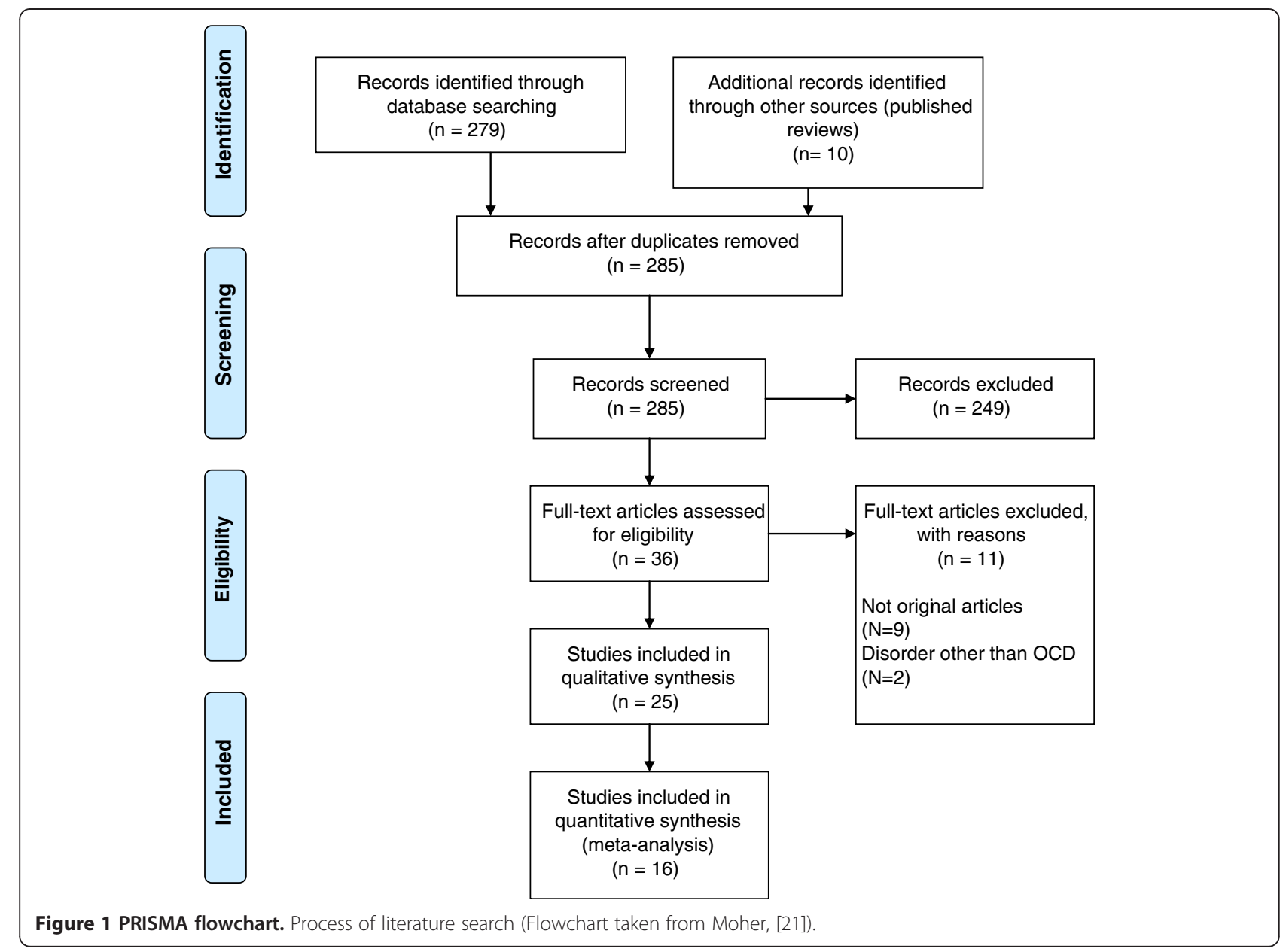

the ALIC in three out of four OCD patients. However, this evaluation is based on clinical observation only; no quantitative data have been reported.

In 2003, Nuttin et al. improved on the assessment procedure and made use of a blinded crossover design with alternating stimulation on/off-periods of 3 months with four OCD patients. The Y-BOCS scores were significantly lower at the end of the active stimulation compared to sham stimulation (placebo) $(19.8 \pm 8.0 \mathrm{vs}$. $32.3 \pm 3.9$ ). In an addendum to the main text, information on four additional patients is provided. Y-BOCS reductions are above $35 \%$ for four out of six patients. One patient, for whom DBS did not proof effective, received capsulotomy. For the other two patients only an anecdotal report is available [23].

The same group [24] reported three cases of OCD patients receiving DBS of the ALIC, one of them was already included in Nuttin et al.'s [13] study. In one case, electrodes were removed 15 months after implantation, for the remaining two patients Y-BOCS scores dropped 12 and 23 points from baseline. The latest follow up was 33 and 39 months after surgery [24].
A single case of ALIC DBS was reported by Anderson and Ahmed in 2003. The latest follow-up was performed three months after surgery, so that the Y-BOCS reduction of $79.4 \%$ (from 34 to 7 points) needs to be regarded with caution [25].

Y-BOCS scores of four patients undergoing bilateral ALIC stimulation were assessed in a short-term, blinded, on-off design with four consecutive 3 week periods, as well as in an unblinded, long-term follow up ranging from 4 to 23 months (Abelson et al., [26]). The first design revealed mixed results, with two patients reporting lower mean Y-BOCS scores in the off-condition, compared to the on-condition (on vs. off: 37.5 vs. $39,31.5$ vs. 29,10 vs. 23,27 vs. 26 , respectively). In the latter design two of the four patients could be classified as responders, showing a $44 \%$ and $73 \%$ reduction from baseline, respectively. The other two patients showed a $0 \%$ and $4 \%$ reduction, respectively.

Servello et al. [27] reported thalamic DBS in four patients with Tourette syndrome and comorbid OCD. Case one did not show significant improvement in Y-BOCS scores 30 months after DBS with scores remaining 
around the baseline level of 23 points. Also an additional surgery to implant rescue leads into ALIC/NAc region did not result in a significant symptom reduction. The latest follow-up was performed 44 months after the first surgery. Also the second patient received a "rescue surgery" ten months after electrode implantation. In the 20 months follow-up period Y-BOCS scores improved only mildly with a drop by 13 points from baseline at 18 months post-surgery (from 38 to 25) representing the biggest symptom reduction. Case three improved progressively from a Y-BOCS score of 38 pre-surgery to 15 at the 19 months follow up. The fourth patient displayed a similar improvement (16 vs. 35) in a period of 10 months [27] (see Table 1).

\section{Nucleus accumbens}

As a component of the ventral striatum, the NAc is located beneath the ALIC, at the junction between the head of the caudate and the anterior portion of the putamen. Due to the spatial proximity of NAc and ALIC it is possible to stimulate both target structures. By placing the tip of the electrode in the NAc the deepest contact will stimulate the ventral striatal region, whereas the most dorsal contact, towards the top of the electrode might affect also the ALIC [28]. The NAc, the main gate structure of the basal ganglia is considered to be a motor-limbic interface [29] associated with reward processing, which makes it a promising target structure for many psychiatric disorders and OCD in particular, since a dysfunction in the reward system, i.e. the hyperactivity between NAc and prefrontal cortex, may be an underlying cause for the disorder.

In 2003, Sturm et al. [30] conducted a pilot study with four patients suffering from anxiety and OCD. Authors report total recovery from anxiety- and OCD-symptoms in all but one patient, but no Y-BOCS scores have been reported [30], therefore an investigator bias cannot be excluded. Based on these findings, a larger, methodologically improved study (including a brief double blind crossover phase) was conducted. The results of this study published in 2010, put the previous, overly positive results in perspective. In this study conducted by Huff et al. [31] ten patients received stimulation but unilateral to the right NAc. Five patients were classified as partial responders (i.e., a minimal symptom reduction 25\%) and one patient as a full responder (i.e., a minimal symptom reduction of 35\%). Mean Y-BOCS scores indicated a significant decrease of OCD symptoms after 12 months of active stimulation (from $32.2 \pm 4.0$ to $25.4 \pm 6.7$ ).

A single case of DBS targeting the ventral caudate nucleus in a patient suffering from severe OCD and concomitant major depression was published by Aouizerate et al. [32]. The baseline Y-BOCS score of 25 did not change significantly in the first 9 months of stimulation, but decreased dramatically to 10 and 14 points 12 and 15 months after surgery [32].

Plewnia et al. [33] reported on the single case of a woman suffering from OCD, as well as residual symptoms of schizophrenia, who received DBS of the right NAc. Y-BOCS scores improved from 32 points one month prior to surgery to 24 four weeks after treatment onset and remained at this level for the two year follow up period.

Denys and colleagues published data on 16 OCD patients with DBS of the NAc. After eight months open phase, Y-BOCS scores decreased by $46 \%$. Nine of the patients were classified as responders. Following the open phase period the authors conducted a doubleblind two week cross-over design, of active or sham stimulation, which underlined the positive treatment effects [12]. It is noteworthy that in this study an additional treatment of cognitive behavioral therapy (CBT) was offered once a substantial decrease in Y-BOCS scores (six points on average) was achieved by DBS. In accordance with the therapy refractoriness criteria, CBT was not effective prior to surgery [34]. However, in combination with DBS a substantial improvement in CBT efficacy was reported. This may indicate that DBS creates the necessary readiness of the brain to implement elements of CBT.

Table 1 Trials targeting the ALIC

\begin{tabular}{lcccc}
\hline ALIC & & & & \\
\hline Reference & Responders (ratio) & Follow-up & Patients included elsewhere & Y-BOCS Reduction \\
\hline Gabriels, 2003 [24] & $2 / 3$ & 33 months & 1 patient included in Nuttin, [13] & $35.5 \%$ \\
Anderson \& Ahmed, 2003 [25] & $1 / 1$ & 3 months & & $79.4 \%$ \\
Nuttin, 2003* [23] & $3 / 6$ & 31 months & 3 patients included in Gabriels, [24] & $17.6-72 \%$ \\
Abelson, 2005* [26] & $2 / 4$ & 23 months & & $0-73.3 \%$ \\
Servello, 2009* [27] & $2 / 4$ & 51 months & & $8.7-60.5 \%$ \\
Mean & $\mathbf{7 5 \%}$ & & & $\mathbf{4 6 . 5 \%}$
\end{tabular}

ALIC: anterior limb of the internal capsule. Responders' ratio: responders defined as 35\% improvement in Y-BOCS score or more. Follow-up: latest follow-up reported. Patients included elsewhere: Number of patients of which the data has been reported before and corresponding publication. Y-BOCS reduction: mean reduction for new patients at latest follow-up or reduction range if individual data not available. ${ }^{*}$ Data not included in pooled means due to missing information/republished data. 
Franzini et al. [35] describe two cases of NAc DBS in OCD patients. Patient one was additionally diagnosed with comorbid bipolar disorder type I. His Y-BOCS scores improved from 38 to 22 at the 24 months followup. Patient two was diagnosed with comorbid body dysmorphic disorder, phobic anxiety disorder and depressive disorder, 27 months after surgery he showed a 10 point decrease (from 30 to 20) in Y-BOCS scores [35].

Roh et al. [36] described a further DBS study targeting the NAc with the proximal contact extending into part of the caudate nucleus and the IC in four OCD patients. After 24 months of treatment, baseline Y-BOCS scores decreased from an average $37.0 \pm 1.8$ to $14.8 \pm 5.0$, corresponding to an improvement rate of $59.7 \pm 14.6 \%$ [36].

Barcia et al. [37] used the possibility of intra-individual comparison between two stimulation targets (NAc and STN) in two patients. Different combinations of electrodes were activated for two days, followed by a oneday washout period. Bilateral stimulation of the NAc led to a decrease in Y-BOCS score from 33 to 20 for patient 1 and from 33 to 16 for patient 2. Stimulation of the left accumbens and left STN yielded the lowest Y-BOCS scores for both patients (patient 1: 16; patient 2: 12) (data for STN-DBS can be found under 3.4). Unfortunately, one has to be cautious to conjecture interpretations due to the very low number of subjects [37] (see Table 2).

\section{Ventral Capsule/Ventral Striatum (VC/VS)}

The ventral striatal (VS) area encompasses the ventral caudate nucleus and nucleus accumbens and is thought to be involved in motivation and reward. Combined with the ventral capsule (VC), it is referred to as the VC/VS region.

Greenberg et al. [39] conducted a study with bilateral stimulation of the VC/VS in $10 \mathrm{OCD}$ patients. They administered follow-ups at 1, 3, 6, 12, 18, 24, 30 and 36 months after surgery, the follow-up at 36 months did only capture 8 patients (one patient died 9 months after surgery, due to cancer and her data has not been included, and one patient dropped out after 24 months.) Y-BOCS scores improved from $34.6 \pm 0.6$ (mean \pm SEM) pre-operatively to $25 \pm 1.6$ at 3 months and $22.3 \pm 2.1$ at 36 months [39].

In 2010 Greenberg et al. published results of 26 patients who underwent bilateral DBS of the VC/VS divided over four different research centers, including 10 patients they had previously reported. Follow-up periods in these studies ranged from 3 to 36 months, averaging 31.4 months. Y-BOCS scores improved from $34.0 \pm 0.5$ at baseline to $20.9 \pm 2.4$ at the latest follow-up, with this level of improvement being apparent already 3 months after active stimulation started (Y-BOCS $21.0 \pm 1.8$ ). Furthermore, the authors note that about $65 \%$ of the patients benefitted from the treatment due to clinically significant symptom reductions and functional improvement [40].

Six patients who received bilateral DBS of the VC/VS were assessed in a randomized, blinded, staggered onset design [41]. After 12 months of active stimulation $67 \%$ (i.e. four out of six) patients were classified as responders ( $\geq 35 \%$ Y-BOCS score decrease from baseline). In fact, the mean baseline Y-BOCS score of 33.7 decreased by $15.67 \pm$ 11.60 points (mean \pm SD).

In another study targeting the VC/VS bilaterally, four OCD patients were assessed every 3 months for 15 months showing a $33.6 \%$ reduction (mean $\pm \mathrm{SD}, 36.3 \pm 2.1$ preoperatively vs. $24.3 \pm 9.1$ at 15 months) [42].

In 2013 a single case report of a 51-Year old OCD patient who received DBS of the VC/VS was published. Her Y-BOCS scores fluctuated during the course of her 22-month follow-up, remaining somewhat stagnant over the first 9 months and then decreasing more dramatically to 10 points at 12 months follow-up. However, concomitant psychotherapy makes it difficult to attribute changes to DBS alone [38] (see Table 3).

Table 2 Trials targeting the NAc region

\begin{tabular}{|c|c|c|c|c|}
\hline \multicolumn{5}{|l|}{ NAc } \\
\hline Reference & Responders (ratio) & Follow-up & Patients included elsewhere & Y-BOCS Reduction \\
\hline Aouizerate, 2004 [32] & $1 / 1$ & 15 months & & $52 \%$ \\
\hline Plewnia, 2008 [33] & $0 / 1$ & 24 months & & $21.9 \%$ \\
\hline Denys, 2010 [12] & $9 / 16$ & 8 months & & $46 \%$ \\
\hline Franzini, 2010 [35] & $1 / 2$ & 24 months & & $38.2 \%$ \\
\hline Huff, 2010 [31] & $1 / 10$ & 12 months & & $21.1 \%$ \\
\hline Roh, 2012* [36] & $4 / 4$ & 24 months & & $45.7-79.5 \%$ \\
\hline Barcia, 2013 [37] & $2 / 2$ & 3 months & & $43.9 \%$ \\
\hline Corveleyn, 2013 [38] & $1 / 1$ & 22 months & & $63.9 \%$ \\
\hline Mean & $45.5 \%$ & & & $37.8 \%$ \\
\hline
\end{tabular}

NAc: Nucleus accumbens. Responders' ratio: responders defined as 35\% improvement in Y-BOCS score or more. Follow-up: latest follow-up reported. Patients included elsewhere: Data Number of patients of which the data has been reported before and corresponding publication. Y-BOCS reduction: mean reduction for new patients at latest follow-up or reduction range if individual data not available. *Data not included in pooled means due to missing information. 
Table 3 Trials targeting the VC/VS region

\begin{tabular}{|c|c|c|c|c|}
\hline VC/VS & & & & \\
\hline Reference & Responders (ratio) & Follow-up & Patients published elsewhere & Y-BOCS Reduction \\
\hline Greenberg, 2006 [39] & $4 / 8$ & 36 months & & $35.5 \%$ \\
\hline Greenberg, 2010* [40] & $16 / 26$ & 36 months & $\begin{array}{l}10 \text { patients from Greenberg et al. [39], and } \\
\text { five patients included in Nuttin et al. [23] }\end{array}$ & $38.5 \%$ \\
\hline Goodman, 2010* [41] & $4 / 6$ & 12 months & Five patients from Greenberg et al. [39] & $91.3 \%$ \\
\hline Tsai, 2012 [42] & $2 / 4$ & 15 months & & $53,4 \%$ \\
\hline Mean & $50 \%$ & & & $41.5 \%$ \\
\hline
\end{tabular}

VC/VS: ventral capsule/ventral striatum. Responders' ratio: responders defined as 35\% improvement in Y-BOCS score or more. Follow-up: latest follow-up reported. Patients included elsewhere: Number of patients of which the data has been reported before and corresponding publication. Y-BOCS reduction: mean reduction for new patients at latest follow-up or reduction range if individual data not available. *Data not included in pooled means due to missing information/republisheded data.

\section{Subthalamic nucleus}

The STN is a component of the basal ganglia and is located ventral to the thalamus, dorsal to the substantia nigra, and medial to the corticospinal tract. Experiences with DBS in patients with Parkinson's disease (PD) and comorbid OCD symptoms have suggested the involvement of the STN in OCD.

Mallet et al. [43] described a successful stimulation of the STN in two OCD patients with improvements of 58 and 64\% (pre-operative Y-BOCS 26 and 23, respectively; Y-BOCS at 6 months follow-up 5 and 4, respectively) [43]. Fontaine et al. [44] reported a second positive case: A 49-year-old man suffering from OCD as well as PD received bilateral stimulation of the STN, reducing YBOCS scores from a baseline of 32 to 1 after 6 months of active stimulation [44].

Six years after their first report on STN DBS in OCD, Mallet et al. [45] published a study with a double-blind crossover design by which they assess the efficacy of bilateral STN stimulation. Sixteen OCD patients were randomly assigned to active stimulation followed by sham stimulation, or vice versa, with eight patients in each group. Assessment at the end of the two 3 months periods revealed a significantly lower Y-BOCS score for patients under active stimulation (mean $\pm \mathrm{SD}, 19 \pm 8$ ) compared to patients under sham stimulation (28 \pm 7 ) [45].

Finally, Chabardès et al. [46] described four patients, two of them originally reported by Mallet et al. [45]. Of the remaining two, one showed a symptom reduction of $78 \%$ (8 vs. 29 ) while the second patient was classified as a partial responder with an improvement of about $34 \%$ (21 vs. 32) 6 months after electrode implantation [46]. STN-DBS data of the study by Barcia et al. [37] revealed the following improvement in Y-BOCS scores: scores of patient 1 decreased from 33 at baseline to 20 three months follow-up and of patient 2 decreased from 33 at baseline to 18 (see Table 4).

\section{Inferior thalamic peduncle}

The ITP is the connecting structure between orbitofrontal cortex and thalamus and part of the orbitofrontal thalamic system. Its association with OCD symptoms makes it a promising target structure for DBS.

In 2009 Jiménez et al. reported bilateral ITP stimulation in five patients with refractory OCD. Y-BOCS scores were assessed every 3 months for a total of 12 months, showing a reduction of $51 \%$ at the last follow-up (mean \pm SD, $17.8 \pm 3.9$ vs. $35 \pm 6.2$,) [47]

Another study by Jiménez et al. [48] assessed the efficacy of implantation of quadripolar deep brain stimulation electrodes in the ITP for six OCD patients. At 12 months follow-up a $51 \%$ decrease in Y-BOCS scores could be observed (mean \pm SD, $17.5 \pm 3.6$ vs. $35.8 \pm 5.9$ ) which further decreased after 24 and 36 months (14.0 \pm 7. 8 and 13.3 \pm 5.7 , respectively) [48] (see Table 5).

\section{Adverse events}

Complications of DBS can be classified into 3 categories. First, 'procedure related adverse events' describe complications arising from the surgery itself. Second, difficulties may originate from the implantation device ('device related'). Third, the stimulation itself may cause effects on mood or cognition that can be acute and reversible or chronic in nature and are specific to the target region [40]. Disorder related adverse events are not discussed at this point.

\section{Procedure related}

Overall, six patients suffered from superficial wound infections following surgery $[39,40]$ and one patient exhibited an allergic reaction to the implantation device in the chest [42]. Dell'Osso and colleagues described a patient who, following a minor inflammation at the area where the stimulator had been implanted, developed a scar-picking behavior, making it necessary to remove the device despite major improvements in OCD symptoms [49].

Serious adverse events during surgery included two seizures $[39,40]$, and three intracerebral hemorrhages $[39,40,45]$. 
Table 4 Trials targeting the STN region

\begin{tabular}{|c|c|c|c|c|}
\hline \multicolumn{5}{|l|}{ STN } \\
\hline Reference & Responders (ratio) & Follow-up & Patients included elsewhere & Y-BOCS Reduction \\
\hline Mallet, 2002 [43] & $2 / 2$ & 6 months & & $82.7 \%$ \\
\hline Fontaine, 2004 [44] & $1 / 1$ & 6 months & & $96.8 \%$ \\
\hline Mallet, 2008 [45] & $7 / 16$ & 3 months & & $37.8 \%$ \\
\hline Chabardès, 2012* [46] & $2 / 4$ & 6 months & two patients from Mallet et al. [45] & $53.4 \%$ \\
\hline Barcia, 2013 [37] & $2 / 2$ & 3 months & & $42.5 \%$ \\
\hline Mean & $57.1 \%$ & & & $45.3 \%$ \\
\hline
\end{tabular}

\section{Device related}

In five patients breaks in stimulating leads or extension wires requiring replacement were reported $[26,40]$. One patient reported dysesthesia in the subclavicular region that lasted for several weeks [31]. Okun and colleagues reviewed symptoms potentially related to battery failure in 6 patients with refractory OCD, noting that suicidality, mood disturbance, panic attacks, fatigue, and a restless sensation in the extremities may be associated with battery failure [50]. Nuttin et al. [23], as well as Denys and colleagues [12] reported on several patients who felt the leads or stimulation devices (5 permanent and 7 transient cases).

\section{Stimulation related}

Anxiety Twenty-five patients in four studies (three NAc studies, one VC/VS study) $[30,31,36,40]$ experienced an acute increase in anxiety. All of these were induced either by a change of stimulation parameters, or by battery depletion, so that anxiety symptoms were quickly resolved after parameter adaption or pacemaker exchange.

Mood The most common effect on mood during chronic DBS was hypomania, experienced by 27 patients across five studies (two NAc studies and three VC/VS studies) [31,36,40-42]. In all cases mood elevation responded to parameter adjustment. One patient exhibited hypomanialike symptoms during initial DBS programming [42]. Furthermore, Greenberg et al. [40] reported irritability leading to domestic problems in one patient. Increased depression and suicidal thoughts were noted in six patients across three studies (one ALIC study, one NAc study, and one VC/VS study) $[26,31,40]$. It should be noted, however, that some of these patients reported similar episodes prior to surgery. One patient in the study by Abelson et al. [26] whose symptoms were reduced after DBS, committed suicide one year after surgery and stated in a note that her suicide was unrelated to the study.

Cognition Effects of DBS on cognition in patients suffering from OCD seem to occur only rarely. Some patients reported cognitive 'clouding' or diminished concentration in two studies $[31,40]$. However, these were related only to certain parameter settings, and thus readily reversible. In the same study activation of the most dorsal contact during operation resulted in verbal perseveration in one patient. Three subjects in a study by Denys et al. [12] reported words-finding difficulties. In eight patients across two studies $[23,40]$ DBS affected memory performance: one patient had brief flashbacks that recurred several times a day, which could be resolved with changing stimulation parameters [40], the memory problems in the other seven patients were transient and resolved over time [23].

\section{Other}

Next to these psychological effects some authors noted various other effects that may be attributed to DBS. These range from difficulties falling asleep [41] over vertigo [42] and weight loss or gain to long-lasting fatigue

Table 5 Trials targeting the IPT

\begin{tabular}{lclcc}
\hline IPT & & & & \\
\hline Reference & Responders (ratio) & Follow-up & Patients included elsewhere & Y-BOCS Reduction \\
\hline Jimenez, 2009* [47] & $5 / 5$ & 12 months & & $44-58.3 \%$ \\
Jimenez, 2013 [48] & $6 / 6$ & 36 months & 5 patients included in Jimenez 2009 & $82.5 \%$ \\
Mean & $\mathbf{1 0 0 \%}$ & & & $\mathbf{8 2 . 5 \%}$ \\
\hline
\end{tabular}

IPT: Inferior thalamic peduncle. Responders' ratio: responders defined as 35\% improvement in Y-BOCS score or more. Follow-up: latest follow-up reported. Patients included elsewhere: Number of patients of which the data has been reported before and corresponding publication. Y-BOCS reduction: mean reduction for new patients at latest follow-up or reduction range if individual data not available. *Data not include in pooled means due to republished data. 
[23]. Also an increased headache frequency [31,34] and a single case of a visual disturbance in the left eye [37] have been noted.

\section{Discussion}

In 2009 the FDA (under the human device exemption) and the European authorities approved DBS of striatal areas for tr-OCD and thus tr-OCD became the first psychiatric indication approved for DBS treatment. Nevertheless this decision was based on sparse data available at the time. Therefore it is necessary to provide continually a current review on recent clinical studies in order to evaluate the risk-benefit ratio. This was the aim of the present article.

In the scope of reviewing the existing literature it became apparent that three main DBS target regions in the brain are used for tr-OCD. Although publications refer to five different regions: the Nucleus subthalamicus (STN), the inferior thalamic peduncle (ITP), the Nucleus accumbens (NAc), the anterior limb of the internal capsule (ALIC), and the ventral capsule/ventral striatal (VC/VS) area, in our view, the three latter structures refer to one superordinate brain region (Figure 2). In these three brain regions, the electrical field of stimulation might capture portions of the NAc as well as portions of the capsula interna. Further parts of the bed nucleus of the stria terminalis might also be stimulated [40]. Still it should be the aim of future studies to define the best localization within that target area. However pooling the data for these three structures, (ALIC, NAc and VC/VS) the mean response rate is $49 \%$ and the mean Y-BOCS reduction is $39.4 \%$, similar to STN data (57\% responders, $49 \%$ Y-BOCS reduction) On the basis of the reviewed data, DBS of all discussed targets seems to be a promising treatment option for tr-OCD. Procedure as well as device related adverse events are relatively low. Stimulation or therapy related side effects are mostly transient and decline after stimulation parameters have been changed. As mentioned above one person died after DBS surgery and during DBS treatment, but authors stated that death was cancer related. Although an ethical point of discussion is always the question whether DBS affects personality, we could not find any report on personality changes in tr-OCD patients treated with DBS. Although this aspect is difficult to evaluate and should be studied more extensively in future studies [51].

Comparing all available studies, no target structure could be identified that is superior to other structures in terms of efficacy. The response rate for ITP-DBS seems to be higher but is based on only six patients from one research group, on the other hand the available data for NAc-DBS are larger and therefore more consistent. The most relevant problem is that large clinical trials are missing which might be due to the characteristics of the cohort and the fact that treatment resistance needs to be proven [52]. But at least in terms of methodology, studies should become more standardized. Different methodologies in terms of study design as well as several target structures and stimulation parameters make a reliable comparison of data almost impossible. For example, there should be a consensus on definitions of treatment response, remission and recovery. Furthermore, the Y-BOCS is the most common used outcome measure for studies including OCD patients, but some studies do not report these scores, instead subjective statements of the patient. The Y-BOCS by itself is not sufficient since it fails to detect changes in severely affected patients. To complete the picture, quality of life scores should also be obtained, as well as the level of functioning. In any case it is of most relevance to develop a standard design and outcome measure for clinical trials that would make comparison feasible.

Beside the search for the optimal target structure and improvement of response prediction, it is of high relevance to analyze the exact network effects that underlie clinically effective DBS, to understand the neurobiological mechanisms. For this purpose imaging techniques, especially tractography, would be of high importance. Recently,
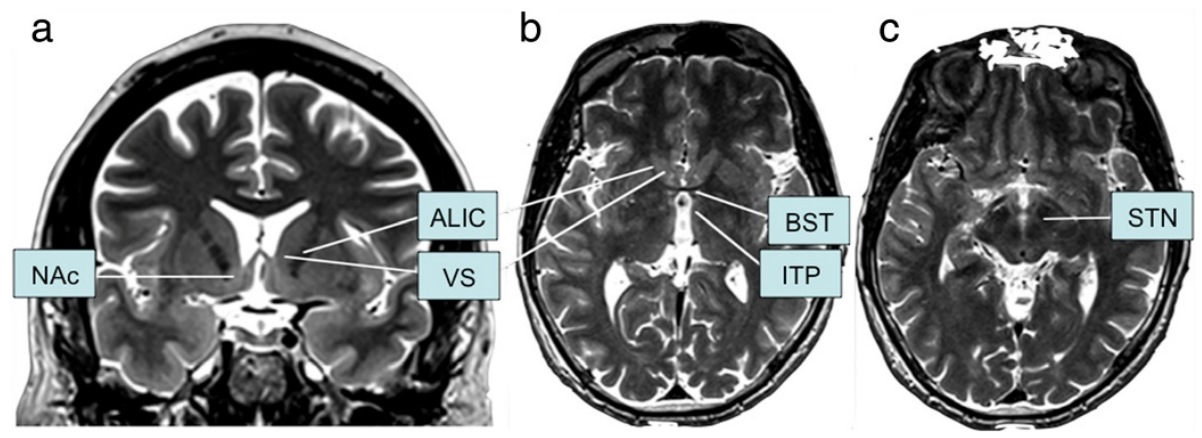

Figure 2 Target locations. Reconstructed targets onto coronal (a) and horizontal (b, c) sections. NAc: Nucleus accumbens; ALIC: Anterior limb of the internal capsule; VS: ventral striatum; BST: Bed nucleus of the stria terminalis; ITP: Inferior thalamic peduncle; STN: subthalamic nucleus. 
research regarding the treatment of depression with DBS of the CG25 suggests, that not a down regulation of the CG25 itself but rather the modulation of white matter tracks is the effective mechanism of action [53]. Also specific electrophysiological data can contribute to decoding the mechanisms.

\section{Conclusion}

Remarkably, we found little difference between anatomical structures in terms of response rates. Stimulation of the STN as well as the ventral striatal structures resulted in mean symptom reductions around $40 \%$, the latter might be associated with slightly more side effects. The reported response rate and symptom reduction after ITP DBS is higher, however, this is based only on the data of six patients by one research group which calls for a critical appraisal.

Future research is strongly needed and clinical trials should focus on optimizing DBS therapy with respect to patient selection and postoperative management, specifically with regard to combining DBS with adjacent cognitive behavioral therapy [34]. Further basic scientific research is also indispensable in order to investigate the mechanisms of action underlying DBS treatment.

\section{Abbreviations}

OCD: Obsessive compulsive disorder; DBS: Deep brain stimulation; tr-OCD: Treatment refractory obsessive compulsive disorder; OFC: Orbitofrontal cortex; ALIC: The anterior limb of the internal capsule; NAc: Nucleus accumbens; VCNS: Ventral capsule/ventral striatum; STN: Subthalamic nucleus; ITP: Inferior thalamic peduncle; Y-BOCS: Yale-Brown Obsessive-Compulsive Scale.

\section{Competing interest}

Jens Kuhn has received financial support for IIT-studies from Medtronic EuropeSARL (Meerbusch, Germany). All other authors declare that they have no competing interest.

\section{Authors' contributions}

SK and DS participated in conducting the PubMed search and reviewing the abstracts. $J \mathrm{~L}$ reviewed the existing literature and completed the list of studies. DD, MU, DL, and W helped writing the manuscript. JK conceived of the study, and participated in its design and coordination and helped to draft the manuscript. All authors read and approved the final manuscript.

\section{Acknowledgment}

We would like to thank the Walter and Marga Boll foundation as well as German Research Foundation (KFO-219 grant) for financial support. The funders had no role in study design, data collection and analysis, decision to publish, or preparation of the manuscript.

\footnotetext{
Author details

${ }^{1}$ Department of Psychiatry and Psychotherapy, University of Cologne, Kerpener Strasse 62, 50937 Cologne, Germany. 'Department of Psychiatry, Academic Medical Center, Meibergdreef 5, 1105 AZ Amsterdam, the Netherlands. ${ }^{3}$ The Netherlands Institute for Neuroscience, an institute of the Royal Netherlands Academy of Arts and Sciences, Meibergdreef 47, 1105 BA Amsterdam, the Netherlands. ${ }^{4}$ Department of Psychiatry and Psychotherapy, University of Magdeburg, Leipzigerstrasse 44, 39120 Magdeburg, Germany. ${ }^{5}$ Department of Stereotactic and Functional Neurosurgery, University of Cologne, Kerpener Strasse 62, 50937 Cologne, Germany.
}

Received: 24 March 2014 Accepted: 18 July 2014

Published: 2 August 2014

\section{References}

1. Murray CJ, Lopez AD: Evidence-based health policy-lessons from the Global Burden of Disease Study. Science 1996, 274(5288):740-743.

2. Robins LN, Helzer JE, Weissman MM, Orvaschel H, Gruenberg E, Burke JD Jr, Regier DA: Lifetime prevalence of specific psychiatric disorders in three sites. Arch Gen Psychiatry 1984, 41(10):949-958.

3. Mclntyre CC, Hahn PJ: Network perspectives on the mechanisms of deep brain stimulation. Neurobiol Dis 2010, 38(3):329-337.

4. Modell JG, Mountz JM, Curtis GC, Greden JF: Neurophysiologic dysfunction in basal ganglia/limbic striatal and thalamocortical circuits as a pathogenetic mechanism of obsessive-compulsive disorder. J Neuropsychiatry Clin Neurosci 1989, 1(1):27-36.

5. Beucke JC, Sepulcre J, Talukdar T, Linnman C, Zschenderlein K, Endrass T, Kaufmann C, Kathmann N: Abnormally high degree connectivity of the orbitofrontal cortex in obsessive-compulsive disorder. JAMA Psychiatry 2013, 70(6):619-629.

6. Ahmari SE, Spellman T, Douglass NL, Kheirbek MA, Simpson HB, Deisseroth K, Gordon JA, Hen R: Repeated cortico-striatal stimulation generates persistent OCD-like behavior. Science 2013, 340(6137):1234-1239.

7. Denys D: Pharmacotherapy of obsessive-compulsive disorder and obsessive-compulsive spectrum disorders. Psychiatr Clin North Am 2006, 29(2):553-584. xi.

8. Benabid AL, Pollak P, Louveau A, Henry S, de Rougemont J: Combined (thalamotomy and stimulation) stereotactic surgery of the VIM thalamic nucleus for bilateral Parkinson disease. Appl Neurophysiol 1987, 50(1-6):344-346.

9. Kuhn J, Grundler TO, Lenartz D, Sturm V, Klosterkotter J, Huff W: Deep brain stimulation for psychiatric disorders. Dtsch Arztebl Int 2010, 107(7):105-113.

10. McCracken $C B$, Grace $A A$ : Nucleus accumbens deep brain stimulation produces region-specific alterations in local field potential oscillations and evoked responses in vivo. J Neurosci 2009, 29(16):5354-5363.

11. Dell'Osso B, Altamura AC, Allen A, Hollander E: Brain stimulation techniques in the treatment of obsessive-compulsive disorder: current and future directions. CNS Spectr 2005, 10(12):966-979. 983.

12. Denys $D$, Mantione $M$, Figee $M$, van den Munckhof $P$, Koerselman $F$, Westenberg H, Bosch A, Schuurman R: Deep brain stimulation of the nucleus accumbens for treatment-refractory obsessive-compulsive disorder. Arch Gen Psychiatry 2010, 67(10):1061-1068.

13. Nuttin B, Cosyns P, Demeulemeester H, Gybels J, Meyerson B: Electrical stimulation in anterior limbs of internal capsules in patients with obsessive-compulsive disorder. Lancet 1999, 354(9189):1526.

14. Kuhn J, Lenartz D, Mai JK, Huff W, Lee SH, Koulousakis A, Klosterkoetter J, Sturm $\vee$ : Deep brain stimulation of the nucleus accumbens and the internal capsule in therapeutically refractory Tourette-syndrome. J Neurol 2007, 254(7):963-965.

15. Vandewalle V, van der Linden C, Groenewegen HJ, Caemaert J: Stereotactic treatment of Gilles de la Tourette syndrome by high frequency stimulation of thalamus. Lancet 1999, 353(9154):724.

16. Kuhn J, Lenartz D, Huff W, Lee S, Koulousakis A, Klosterkoetter J, Sturm V: Remission of alcohol dependency following deep brain stimulation of the nucleus accumbens: valuable therapeutic implications? J Neurol Neurosurg Psychiatry 2007, 78(10):1152-1153.

17. Muller UJ, Voges J, Steiner J, Galazky I, Heinze HJ, Moller M, Pisapia J, Halpern C, Caplan A, Bogerts B, Kuhn J: Deep brain stimulation of the nucleus accumbens for the treatment of addiction. Ann N Y Acad Sci 2013, 1282:119-128.

18. Mayberg HS, Lozano AM, Voon V, McNeely HE, Seminowicz D, Hamani C, Schwalb JM, Kennedy SH: Deep brain stimulation for treatment-resistant depression. Neuron 2005, 45(5):651-660.

19. Figee M, Luigjes J, Smolders R, Valencia-Alfonso CE, van Wingen $G$, de Kwaasteniet B, Mantione M, Ooms P, de Koning P, Vulink N, Levar N, Droge $L$, van den Munckhof P, Schuurman PR, Nederveen A, van den Brink W, Mazaheri A, Vink M, Denys D: Deep brain stimulation restores frontostriatal network activity in obsessive-compulsive disorder. Nat Neurosci 2013, 16(4):386-387.

20. Luigjes J, de Kwaasteniet BP, de Koning PP, Oudijn MS, van den Munckhof $P$, Schuurman PR, Denys D: Surgery for psychiatric disorders. World Neurosurg 2013, 80(3-4):S31 e17-28. 
21. Moher D, Liberati A, Tetzlaff J, Altman DG: Preferred reporting items for systematic reviews and meta-analyses: the PRISMA statement. PLoS Med 2009, 6(7):e1000097.

22. Mindus $P$, Rasmussen SA, Lindquist C: Neurosurgical treatment for refractory obsessive-compulsive disorder: implications for understanding frontal lobe function. J Neuropsychiatry Clin Neurosci 1994, 6(4):467-477.

23. Nuttin B, Gabriels LA, Cosyns PR, Meyerson BA, Andreewitch S, Sunaert SG, Maes AF, Dupont PJ, Gybels JM, Gielen F, Demeulemeester HG: Long-term electrical capsular stimulation in patients with obsessive-compulsive disorder. Neurosurgery 2003, 52(6):1263-1272. discussion 1272-1264.

24. Gabriels L, Cosyns P, Nuttin B, Demeulemeester H, Gybels J: Deep brain stimulation for treatment-refractory obsessive-compulsive disorder: psychopathological and neuropsychological outcome in three cases. Acta Psychiatr Scand 2003, 107(4):275-282.

25. Anderson D, Ahmed A: Treatment of patients with intractable obsessivecompulsive disorder with anterior capsular stimulation. Case report $J$ Neurosurg 2003, 98(5):1104-1108.

26. Abelson JL, Curtis GC, Sagher O, Albucher RC, Harrigan M, Taylor SF, Martis B, Giordani B: Deep brain stimulation for refractory obsessive-compulsive disorder. Biol Psychiat 2005, 57(5):510-516

27. Servello D, Sassi M, Bastianello S, Poloni GU, Mancini F, Pacchetti C: Electrode displacement after intracerebral hematoma as a complication of a deep brain stimulation procedure. Neuropsychiatr Dis Treat 2009, 5:183-187.

28. Okun MS, Mann G, Foote KD, Shapira NA, Bowers D, Springer U, Knight W Martin P, Goodman WK: Deep brain stimulation in the internal capsule and nucleus accumbens region: responses observed during active and sham programming. J Neurol Neurosurg Psychiatry 2007, 78(3):310-314.

29. Mogenson GJ, Yang CR: The contribution of basal forebrain to limbicmotor integration and the mediation of motivation to action. Adv Exp Med Biol 1991, 295:267-290.

30. Sturm V, Lenartz D, Koulousakis A, Treuer H, Herholz K, Klein JC, Klosterkotter J: The nucleus accumbens: a target for deep brain stimulation in obsessivecompulsive- and anxiety-disorders. J Chem Neuroanat 2003, 26(4):293-299.

31. Huff W, Lenartz D, Schormann M, Lee SH, Kuhn J, Koulousakis A, Mai J, Daumann J, Maarouf M, Klosterkotter J, Sturm V: Unilateral deep brain stimulation of the nucleus accumbens in patients with treatmentresistant obsessive-compulsive disorder: Outcomes after one year. Clin Neurol Neurosurg 2010, 112(2):137-143.

32. Aouizerate B, Cuny E, Martin-Guehl C, Guehl D, Amieva H, Benazzouz A, Fabrigoule C, Allard M, Rougier A, Bioulac B, Tignol J, Burbaud P: Deep brain stimulation of the ventral caudate nucleus in the treatment of obsessive-compulsive disorder and major depression. Case report J Neurosurg 2004, 101(4):682-686

33. Plewnia C, Schober F, Rilk A, Buchkremer G, Reimold M, Wachter T, Breit S, Weiss D, Kruger R, Freudenstein D: Sustained improvement of obsessivecompulsive disorder by deep brain stimulation in a woman with residual schizophrenia. Int J Neuropsychopharmacol 2008, 11(8):1181-1183.

34. Shimizu E: [Definition of treatment-refractory obsessive-compulsive disorder based on cognitive behavioral therapy]. Seishin Shinkeigaku Zasshi 2013, 115(9):975-980.

35. Franzini A, Messina G, Gambini O, Muffatti R, Scarone S, Cordella R, Broggi G: Deep-brain stimulation of the nucleus accumbens in obsessive compulsive disorder: clinical, surgical and electrophysiological considerations in two consecutive patients. Neuro/ Sci 2010, 31(3):353-359.

36. Roh D, Chang WS, Chang JW, Kim CH: Long-term follow-up of deep brain stimulation for refractory obsessive-compulsive disorder. Psychiatry Res 2012, 200(2-3):1067-1070.

37. Barcia JA, Reyes L, Arza R, Saceda J, Avecillas J, Yanez R, Garcia-Albea J, Ortiz T, Lopez-Ibor MI, Lopez-Ibor JJ: Deep Brain Stimulation for ObsessiveCompulsive Disorder: Is the Side Relevant? Stereotact Funct Neurosurg 2013, 92(1):31-36.

38. Corveleyn P, Nuttin B, Gabriels L: Deep brain stimulation in a patient with ocd and the intensive pre- and post-operative psychiatric/psychotherapeutic follow-up. A case study. Tijdschr Psychiatr 2013, 55(3):203-208.

39. Greenberg BD, Malone DA, Friehs GM, Rezai AR, Kubu CS, Malloy PF, Salloway SP, Okun MS, Goodman WK, Rasmussen SA: Three-year outcomes in deep brain stimulation for highly resistant obsessive-compulsive disorder. Neuropsychopharmacology 2006, 31(11):2384-2393.

40. Greenberg BD, Gabriels LA, Malone DA Jr, Rezai AR, Friehs GM, Okun MS, Shapira NA, Foote KD, Cosyns PR, Kubu CS, Malloy PF, Salloway SP, Giftakis JE, Rise MT, Machado AG, Baker KB, Stypulkowski PH, Goodman WK,
Rasmussen SA, Nuttin BJ: Deep brain stimulation of the ventral internal capsule/ventral striatum for obsessive-compulsive disorder: worldwide experience. Mol Psychiatry 2010, 15(1):64-79.

41. Goodman WK, Foote KD, Greenberg BD, Ricciuti N, Bauer R, Ward H, Shapira NA, Wu SS, Hill CL, Rasmussen SA, Okun MS: Deep brain stimulation for intractable obsessive compulsive disorder: pilot study using a blinded, staggered-onset design. Biol Psychiat 2010, 67(6):535-542.

42. Tsai HC, Chang CH, Pan J, Hsieh HJ, Tsai ST, Hung HY, Chen SY: Pilot study of deep brain stimulation in refractory obsessive-compulsive disorder ethnic Chinese patients. Psychiatry Clin Neurosci 2012, 66(4):303-312.

43. Mallet L, Mesnage V, Houeto JL, Pelissolo A, Yelnik J, Behar C, Gargiulo M, Welter ML, Bonnet AM, Pillon B, Cornu P, Dormont D, Pidoux B, Allilaire JF, Agid Y: Compulsions, Parkinson's disease, and stimulation. Lancet 2002, 360(9342):1302-1304

44. Fontaine D, Mattei V, Borg M, von Langsdorff D, Magnie MN, Chanalet S, Robert $P$, Paquis P: Effect of subthalamic nucleus stimulation on obsessive-compulsive disorder in a patient with Parkinson disease. Case report J Neurosurg 2004, 100(6):1084-1086

45. Mallet L, Polosan M, Jaafari N, Baup N, Welter ML, Fontaine D, du Montcel ST, Yelnik J, Chereau I, Arbus C, Raoul S, Aouizerate B, Damier P, Chabardès S, Czernecki V, Ardouin C, Krebs MO, Bardinet E, Chaynes P, Burbaud P, Cornu P, Derost P, Bougerol T, Bataille B, Mattei V, Dormont D, Devaux B, Vérin $M$, Houeto $J$, Pollak $P$, et al: Subthalamic nucleus stimulation in severe obsessive-compulsive disorder. N Engl J Med 2008, 359(20):2121-2134.

46. Chabardes S, Polosan M, Krack P, Bastin J, Krainik A, David O, Bougerol T, Benabid AL: Deep brain stimulation for obsessive-compulsive disorder: subthalamic nucleus target. World Neurosurg 2013, 80(3-4):S31 e31-38.

47. Jimenez-Ponce F, Velasco-Campos F, Castro-Farfan G, Nicolini H, Velasco AL, Salin-Pascual R, Trejo D, Criales JL: Preliminary study in patients with obsessive-compulsive disorder treated with electrical stimulation in the inferior thalamic peduncle. Neurosurgery 2009, 65(6 Suppl):203-209. discussion 209.

48. Jimenez F, Nicolini H, Lozano AM, Piedimonte F, Salin R, Velasco F: Electrical stimulation of the inferior thalamic peduncle in the treatment of major depression and obsessive compulsive disorders. World Neurosurg 2013, 80(3-4):S30 e17-25.

49. Dell'Osso B, Porta M, Servello D, Altamura AC: Deep Brain Stimulation device removal after scar picking behaviors in a patient with treatmentresistant obsessive compulsive disorder. Brain Stimul 2013, 6(1):96-98.

50. Vora AK, Ward H, Foote KD, Goodman WK, Okun MS: Rebound symptoms following battery depletion in the NIH OCD DBS cohort: clinical and reimbursement issues. Brain Stimul 2012, 5(4):599-604.

51. Witt K, Kuhn J, Timmermann L, Zurowski M, Woopen C: Deep Brain Stimulation and the Search for Identity. Neuroethics 2013, 6:499-511.

52. Garnaat SL, Greenberg BD, Sibrava NJ, Goodman WK, Mancebo MC, Eisen JL, Rasmussen SA: Who Qualifies for Deep Brain Stimulation for OCD? Data From a Naturalistic Clinical Sample. J Neuropsychiatry Clin Neurosci 2014, 26(1):81-86

53. Riva-Posse P, Choi KS, Holtzheimer PE, Mclntyre CC, Gross RE, Chaturvedi A, Crowell AL, Garlow SJ, Rajendra JK, Mayberg HS: Defining Critical White Matter Pathways Mediating Successful Subcallosal Cingulate Deep Brain Stimulation for Treatment-Resistant Depression. Biol Psychiat 2014, Advanced online publication. Retrieved from doi:10.1016/j.biopsych.2014.03.029.

\section{doi:10.1186/s12888-014-0214-y}

Cite this article as: Kohl et al.: Deep brain stimulation for treatmentrefractory obsessive compulsive disorder: a systematic review. $B M C$ Psychiatry 2014 14:214 